\title{
Waste Recycling Can Promote Group Living: A cockroach case study
}

\author{
Jan Rychtár ${ }^{1, *}$, Daniel Frynta ${ }^{2}$, Jakub Tomek ${ }^{3}$, Zuzana Varadínová ${ }^{2}$ \\ Cyril Brom ${ }^{3}$
}

\begin{abstract}
Animals live in groups for a wide variety of reasons. The main benefits are related to anti-predator behaviour, foraging, mate finding, and/or reduction of energetic costs. In this paper we present a game-theoretical model that supports the waste recycling hypothesis. This hypothesis posits that the organic waste materials produced by the members of a group represent a valuable resource that is communally inherited and utilized by group members. Under this hypothesis and on the example of cockroaches, we determine evolutionarily stable strategies of social behaviour and quantify conditions on natural parameter values such as food availability under which the group formation is beneficial.
\end{abstract}

Keywords: social behaviour, aggregation, game theory, ESS

\section{Introduction}

Group living - spending substantial amounts of time in close proximity with individuals of the same species - is a common behaviour that can be found across the animal kingdom [10]. Mathematical models of group living are typically based on evaluating the benefits and costs associated with social or solitary ways of life [3]. Krause and Ruxton [10] list seven distinct benefits of group living: (a) anti-predator vigilance, (b) the dilution effect, (c) predator confusion, (d) foraging benefits, (e) finding a mate, (f) conserving heat, and (g) conserving energy. There are also a number of costs associated with living in groups, most notably the following: (a) increased attack rate, (b) kleptoparasitism, (c) reduction in the local food supply, and (d) increased rate of aggression. Since the benefits and the costs depend not only on the individual strategy but also on the strategy adopted by others, game-theory is especially suited to analyze this behaviour [3].

Cockroaches (Blattodea) belong to an ancient insect order [7] of over 4500 species [12]. Most species (for example the ones living in close proximity to humans such as Periplaneta americana and Blattella germanica, but also the free living species such as Eublaberus distanti or Blaberus discoidalis) are social $[1,16]$. Very few species (such as Thanatophyllum akinetum) are known to be strictly solitary [6,14], and some (such as Schultesia lampyridiformis and Schultesia nitor) can even change the levels of sociality during their life [13, 15].

Several hypotheses potentially explaining group living in cockroaches have been already offered. The group living may serve as a protection against predators [5], a protection from isolation syndrome [8], or it can be a way to attract and select mates [17]. Also, the observed

\footnotetext{
${ }^{1}$ Department of Mathematics and Statistics, The University of North Carolina at Greensboro, Greensboro, NC, USA

${ }^{2}$ Department of Zoology, Faculty of Science, Charles University in Prague, Prague, Czech Republic

${ }^{3}$ Faculty of Mathematics and Physics, Charles University in Prague, Prague, Czech Republic

${ }^{*}$ Correspondence: rychtar@uncg.edu
} 
group living may be just a coincidental aggregation in the same habitat (that has the right level of various environmental conditions such as temperature, humidity and darkness) [4].

Here we focus on the waste recycling hypothesis proposed in [2]. The hypothesis states that the waste organic materials (faeces, cast cuticle (exuviae) and dead bodies of the cockroaches) produced by the members of cockroach colony represent a valuable resource that is communally inherited and utilized by the survivors. As already demonstrated in Blattella germanica [9] or Periplaneta americana [11], the waste indeed represents valuable resources not only for the energetic content, but also because of the content of nutrients which can be deficient in the environment, especially proteins and endosymbionts. If the organic waste material stays protected inside the aggregation shelter, it may provide considerable advantage for individuals present in the aggregation. In contrast, outside the colony, the waste is (a) likely randomly dispersed and (b) unlikely found by cockroaches but rather encountered and consumed by other animals (such as ants).

The purpose of this paper is to provide mathematical support for the waste recycling hypothesis. In Section 2 we build the mathematical model that allows us to quantify benefits and costs of group living and compare them to the benefits and costs of living alone. In Section 3 we analyse the model, provide the evolutionarily stable values of sociality and give conditions on natural parameters under which group living can evolve according to our model. Finally, in Section 4 we summarize our findings and provide some discussion and interpretation of our results.

\section{Mathematical Model}

Our aim is to build a model that will allow us to evaluate the net benefits (benefits, $B$, minus costs, $C$ ) of living alone, $E_{a}=B_{a}-C_{a}$, and the net benefits of living in a colony, $E_{c}=B_{c}-C_{c}$. Since the cockroaches compete for a fixed amount of resources, we will assume that the benefits depend on $s$, where $s \in[0,1]$ is the sociality level in the population, i.e. an average proportion of time spent by an individual cockroach in the colony. We will thus evaluate functions $B_{c}=B_{c}(s)$ and $B_{a}=B_{a}(s)$.

We consider the rates of getting the resources (such as energy and nutrients) as the only indicators of benefits. Let $R$ be rate at which resources naturally occur in the environment (outside of the colony), minus the rate at which animals other than cockroaches find and consume them. Let $N$ be the (large) number of cockroaches in the environment. We assume that even a much smaller number of cockroaches is able to find and eat all resources available to them. Hence, the benefit of being alone when vast majority of cockroaches adopts a sociality level $s$ is given by

$$
B_{a}(s)=\frac{R}{N(1-s)}
$$

which corresponds to the fact that only $N(1-s)$ cockroaches are actually searching for the food outside of the colony (and then eating all they can find). We note that since cockroaches cannot realistically be in the colony all the time, $s$ is bounded away from 1 and thus $B_{a}(s)$ is bounded.

We will assume $C_{c}<C_{a}$ which mimics the fact that staying inside the colony is not as costly (in terms of energy and resources) as searching outside of the colony. We will also assume that the current population of cockroaches reached the carrying capacity of the environment and $N$ is thus fixed and that

$$
C_{a}=B_{a}(0)=\frac{R}{N}
$$

This is because costs and benefits must equal (as otherwise we would get a decline in cockroach population if $C_{a}>B_{a}$ or an increase in cockroach population if $C_{a}<B_{a}$, resulting in $C_{a}=B_{a}$ in either case due to changing $N$ in equation (1)). 
In order to evaluate $B_{c}(s)$, we will assume that waste is the only resource that can be eaten inside the colony. We will therefore track $W^{-}$, a rate at which the organic waste matter inside the colony is eaten (by members of the colony), and $W^{+}$, a rate at which the waste is replenished. For simplicity, we will assume that the only way to replenish a waste (either in the form of faeces, cast cuticle or dead bodies) is by eating and since cockroaches can eat waste inside or other resources outside of the colony, we have $W^{+}=W_{c}^{+}+W_{o}^{+}$, where $W_{c}^{+}\left(\right.$or $\left.W_{o}^{+}\right)$is a rate at which the waste matter is replenished after eating inside the colony (or outside of the colony). Assuming that cockroaches will eat everything that is available inside the colony, we get

$$
W_{c}^{+}=W^{-} \cdot \delta \cdot s
$$

where $W^{-}$corresponds to the amount of waste available and thus actually eaten, $\delta \in(0,1)$ is the proportion of nutrients that will eventually turn back into reusable waste and the multiplication by a factor $s$ corresponds to the fact that the waste has to be produced inside of the colony in order to really contribute to the overall reservoir of the waste organic matter. Similarly, we have

$$
W_{o}^{+}=R \cdot \delta \cdot s
$$

where, as in (3), we assume that the cockroaches eat all that is available to them (at rate $R$ ), regardless of the exact number of them (i.e., no factor $(1-s)$ in (4)), but only a fraction $\delta$ will turn back into reusable waste and only if produced inside of the colony (factor $s$ ). In equilibrium point, the cockroaches eat as much waste as they produce which yields $W^{-}=W^{+}$, and thus

$$
\begin{aligned}
W^{-} & =W_{c}^{+}+W_{o}^{+} \\
& =W^{-} \cdot \delta \cdot s+R \cdot \delta \cdot s .
\end{aligned}
$$

Hence,

$$
W^{-}=\frac{R \delta s}{1-\delta s},
$$

and consequently the benefits for a single cockroach (out of $N s$ cockroaches inside of the colony) are given by

$$
B_{c}(s)=\frac{W^{-}}{N s}=\frac{R}{N} \frac{\delta}{1-\delta s} .
$$

To summarize, the payoff function to a cockroach using strategy $s_{i n d} \in[0,1]$ (i.e. stays in the colony for the $s_{i n d}$ fraction of the time) in the population with an average sociality level $s$ is

$$
U\left(s_{i n d}, s\right)=\left(1-s_{i n d}\right)\left(B_{a}(s)-C_{a}\right)+s_{i n d}\left(B_{c}(s)-C_{c}\right) .
$$

\section{Analysis}

We would like to determine an evolutionarily stable level of sociality in the population. In the framework of the model above, it means to find a critical value $s_{c}$ such that

$$
\begin{aligned}
& E_{a}(s)=E_{c}(s), \text { if } s=s_{c} ; \\
& E_{a}(s)>E_{c}(s), \text { if } s>s_{c}, s \approx s_{c} ; \\
& E_{a}(s)<E_{c}(s), \text { if } s<s_{c}, s \approx s_{c} .
\end{aligned}
$$

If (10)-(12) are satisfied, then when a vast majority of a population adopts a sociality level $s_{c}$ and only a tiny fraction adopts a different strategy $s$ (typically $s=0$ or $s=1$ ), the members of the minority do worse and thus a different strategy cannot spread in the population under the influence of natural selection. 


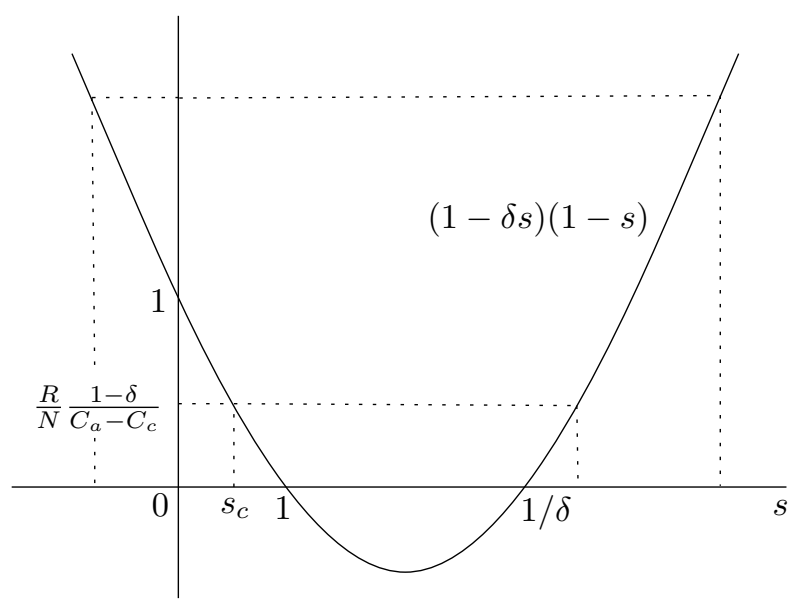

Figure 1: Graph of the function $q(s)=(1-\delta s)(1-s)$ and the two cases of $s_{c}>0$ when $\frac{R}{N} \frac{1-\delta}{C_{a}-C_{c}}<1$ and $s_{c}<0$ when $\frac{R}{N} \frac{1-\delta}{C_{a}-C_{c}}>1$.

Since $E_{c}(s) \gtreqless E_{a}(s)$ if and only if

$$
B_{c}(s)-C_{c} \gtreqless B_{a}(s)-C_{a}
$$

which is true if and only if (according to equations (1) and (8))

$$
(1-\delta s)(1-s) \gtreqless \frac{R}{N} \frac{1-\delta}{C_{a}-C_{c}},
$$

we have to analyze the quadratic function

$$
q(s)=(1-\delta s)(1-s) .
$$

Because $q(1)=0<\frac{R}{N} \frac{1-\delta}{C_{a}-C_{c}}, 0<\delta<1$, and $R>0$, there are two roots of equation $E_{a}(s)=E_{c}(s)$ which corresponds to the quadratic equation

$$
\delta s^{2}-(1+\delta) s+1-\frac{R}{N} \frac{1-\delta}{C_{a}-C_{c}}=0
$$

given by the equality in (14). The larger root of (16) is always bigger than 1 (and thus has no relevance for us), and the smaller root

$$
s_{c}=\frac{(1+\delta)-\sqrt{(1-\delta)^{2}+4 \delta \frac{R}{N} \frac{1-\delta}{C_{a}-C_{c}}}}{2 \delta}
$$

is always smaller than 1 . We know that $s_{c}$ is the point where the left half of the parabola $y=q(s)$ intersects the horizontal line $y=\frac{R}{N} \frac{1-\delta}{C_{a}-C_{c}}$, see Figure 1. Thus, since for $s \in[0,1]$, the function $q(s) \in[0,1]$, we get that $s_{c}>0$ if and only if

$$
q(0)=1>\frac{R}{N} \frac{1-\delta}{C_{a}-C_{c}} .
$$

Because $\frac{d}{d s} q\left(s_{c}\right)<0$ (see also Figure 1), it follows that once (18) holds, $s_{c}$ satisfies (10)-(12) and thus it is evolutionarily stable.

We can see from (17) that $\frac{\partial s_{c}}{\partial R}<0, \frac{\partial s_{c}}{\partial N}>0, \frac{\partial s_{c}}{\partial C_{c}}<0, \frac{\partial s_{c}}{\partial C_{a}}>0$, and $\frac{\partial s_{c}}{\partial \delta}>0$.

Now, we will shift our attention to studying under which conditions the sociality can evolve. Since $\frac{d}{d s} q(s)<0$ for all $s<1$, we get that increasing the level of sociality, $s$, 
decreases the net benefit of being alone, $E_{c}(s)-E_{a}(s)$. Thus, $s=0$ is not stable if and only if $E_{c}(0)-E_{a}(0)<0$, which is by the equivalence between (13) and (14), the same as the condition (18). Consequently, the aggregation behaviour can evolve if and only if (18) holds. Moreover, in a population of asocial cockroaches $(s=0)$ we must have $C_{a}=\frac{R}{N}$ and thus (18) becomes

$$
\delta>\frac{C_{c}}{C_{a}}
$$

\section{Conclusions and Discussion}

In this paper we have built a game theoretical model of group living among cockroaches. Our model supports the waste recycling hypothesis proposed in [2] and it also quantifies the conditions under which the waste organic materials produced by the members of a group can serve as the driving force behind the aggregation behaviour.

We conclude that the group living can evolve from completely solitary behaviour if and only if $\frac{C_{c}}{C_{a}}<\delta$, i.e. if and only if the relative energy expenditures of staying inside compared to the energy costs of searching outside of the group are smaller than the proportion of resources that will be turned into the (reusable) waste. Consequently, aggregation behaviour can evolve if a lot of waste is produced and/or if staying in an aggregation saves a lot of energy.

Once the aggregating behaviour evolved, it can be maintained as long as $1>\frac{R}{N} \frac{1-\delta}{C_{a}-C_{c}}$; in particular if $\frac{R}{N}$, the per capita availability of resources, is small (on top of large $\delta$ and/or small $C_{c} / C_{a}$ as discussed above). Because animals cannot live in environments where the resources are too rare and an excessive amount would be consumed by other animals, the assumption that, in the vicinity of the colony, the resources utilized by cockroaches are moderately rare is reasonable. Also, we have seen that the level of sociality increases as $N$, $\delta$, or $C_{a}$ increases, and the level decreases as $R$ or $C_{c}$ increases.

As any mathematical model, our model has limitations. We have simplified the biological reality. For example, we completely ignored cockroach life stages and did not consider circadian rhythms. We also assumed large population and our reasoning and formulas would not work for small $N$ (as then one cockroach constitutes a significant portion of the population). We did not explicitly model other potential benefits of social living such as protection from the predators (although our model accounted for that by assuming $C_{a}>C_{c}$ ). Finally, the ultimate question is whether our model could be used to validate or invalidate the waste recycling hypothesis experimentally. The parameters such as $C_{c}$ or $C_{a}$, the cost of being in the colony or alone, may be difficult to measured. Yet, our predictions depend on $C_{c} / C_{a}$ and we hope this ratio could be directly or indirectly experimentally gauged and the hypothesis could be proved or disproved.

\section{Acknowledgements}

The research was supported by Simons Foundation grant 245400 .

\section{References}

[1] W. J. Bell, L. M. Roth, and C. A. Nalepa. Cockroaches: ecology, behavior, and natural history. The John Hopkins University Press, Baltimore, MD, USA, 2007.

[2] C. Brom, J. Tomek, J. Rychtář, Z. Varadínová, E. Landová, and D. Frynta. Explaining aggregation behavior of cockroaches by waste recycling hypothesis: mathematical and agent-based models. preprint, in preparation.

[3] M. Broom and J. Rychtáŕ. Game-theoretical Models in Biology. Chapman \& Hall/CRC Press, Boca Raton, FL, 2013. 
[4] M. Dambach and B. Goehlen. Aggregation density and longevity correlate with humidity in first-instar nymphs of the cockroach (Blattella germanica L., Dictyoptera). Journal of Insect Physiology, 45(5):423-429, 1999.

[5] J.-P. Farine, E. Semon, C. Everaerts, D. Abed, P. Grandcolas, and R. Brossut. Defensive secretion of Therea petiveriana: Chemical identification and evidence of an alarm function. Journal of Chemical Ecology, 28(8):1629-1640, 2002.

[6] P. Grandcolas. Is presocial behaviour evolutionarily reversible in cockroaches? Ethology Ecology \&f Evolution, 9(1):69-76, 1997.

[7] D. Grimaldi and M.S. Engel. Evolution of the Insects. Cambridge University Press, 2005.

[8] G. L. Holbrook, E. Armstrong, J. Bachmann, B. M. Deasy, and C. Schal. Role of feeding in the reproductive 'group effect' in females of the German cockroach Blattella germanica (L.). Journal of Insect Physiology, 46(6):941-949, 2000.

[9] R. J. Kopanic, G.L. Holbrook, and V. Sevala. An adaptive benefit of facultative coprophagy in the German cockroach Blattella germanica. Ecological Entomology, 26(2):154-162, 2001.

[10] J. Krause and G. D. Ruxton. Living in groups. Oxford University Press, USA, 2002.

[11] A. Mira. Exuviae eating: a nitrogen meal? Journal of Insect Physiology, 46(4):605-610, 2000 .

[12] L. M. Roth and E. B. Willis. The biotic associations of cockroaches. Smithsonian Miscellaneous Collections, Washington, DC, 1960.

[13] J. van Baaren, A.-S. Bonhomme, P. Deleporte, and J.-S. Pierre. Behaviours promoting grouping or dispersal of mother and neonates in ovoviviparous cockroaches. Insectes Sociaux, 50(1):45-53, 2003.

[14] J. van Baaren, P. Deleporte, P. Grandcolas, V. Biquand, and J.-S. Pierre. Measurement for solitariness and gregarism: analysing spacing, attraction and interactions in four species of Zetoborinae (Blattaria). Ethology, 108(8):697-712, 2002.

[15] J. van Baaren, P. Deleporte, A. Vimard, V. Biquand, and J.-S. Pierre. Weakly aggressive behaviour towards nymphs in the cockroach schultesia nitor (Blattaria: Zetoborinae). Aggressive behavior, 33(6):498-507, 2007.

[16] Z. Varadínová, V. Stejskal, and D. Frynta. Patterns of aggregation behaviour in six species of cockroach: comparing two experimental approaches. Entomologia Experimentalis et Applicata, 136(2):184-190, 2010.

[17] E. Wileyto, G. Boush, and L. M. Gawin. Function of cockroach (Orthoptera: Blattidae) aggregation behavior. Environmental entomology, 13(6):1557-1560, 1984. 